\title{
Reversible Photothermal Homogenization of Fluorous Biphasic Systems with Perfluoroalkylated Nanographene
}

\author{
Guy Guday, a Philip Nickl,a Mohsen Adeli*ab and Rainer Haag*a \\ a Institut für Chemie und Biochemie, Freie Universität Berlin, \\ Takustrasse 3, 14195 Berlin, Germany. \\ b Department of Chemistry, Faculty of Science, Lorestan \\ University, Khorram Abad, 44316-68151 Iran.
}

\section{Corresponding Authors}

^E-mail: haag@chemie.fu-berlin.de (R.H.)

*E-mail: aadeli@zedat.fu-berlin.de (M.A.)

\section{Elemental Analysis Calculations}


$n G \operatorname{Trz}$

Triazine content was calculated based on nitrogen content. $\mathrm{nG}$ with no triazine groups has no measurable nitrogen content, while the triazine functionality conjugated onto graphene will contain 4 nitrogen atoms ( 3 in the ring and one from the nitrene), 3 carbon atoms (all in the ring), and 2 chlorine atoms. It is important to distinguish the carbon content of the Trz groups $\left(\mathrm{C}_{\mathrm{Trz}}\right)$ as compared to that of $\mathrm{nG}\left(\mathrm{C}_{\mathrm{nG}}\right)$ when calculating functionalization density (FD) in terms of number of graphene carbon atoms per functional group. These procedures are shown below, based on the measured results for nGTrz, of $95.84 \mathrm{wt} . \% \mathrm{C}$ and 2.47 wt.\% N. For ease of calculation, a theoretical sample mass of 100 atomic mass units (u).

$$
\begin{gathered}
N=\frac{2.47}{14}=0.17 \\
\operatorname{Tr} Z=\frac{N}{4}=0.044 \\
C_{\operatorname{Trz}}=\operatorname{Tr} z \times 3=0.132 \\
F D=\frac{C_{n G}}{\operatorname{Tr} z \text { groups }}=\frac{\left(\frac{95.84}{12}\right)-0.132}{0.044}=178
\end{gathered}
$$

$n G T r z-P F$

Upon perfluoroalkylation, PF content was calculated based on the sulfur content, assuming that newly added sulfur arises from the thioether bond between PF and Trz. The theoretically expected carbon content from nGTrz was determined by direct normalization of the value from nGTrz (above) based on the nitrogen content in nGTrz-PF, and the total carbon content of nGTrz-PF compared with this value and the expected carbon content from the PF chains, as determined from Sulfur. Similarly, the expected fluorine content of PF was compared against the mass not accounted for by carbon, hydrogen, nitrogen, and sulfur - again, normalized to remove any undetermined mass that would arise from the nGTrz component of the system. As above, a theoretical sample mass of $100 \mathrm{u}$ was used for ease of calculation.

$$
\begin{gathered}
P F=S_{n G T r z-P F}-S_{n G T r z}=\frac{1.85-0.05}{32}=0.056 \\
C_{P F}=P F \times 8=0.45(=5.4 w t . \%) \\
F_{P F}=P F \times 13=0.73(=13.8 w t . \%) \\
C_{n G T r z}=\frac{95.84}{12} \times \frac{1.91}{2.47}=6.18(=74.1 w t . \%)
\end{gathered}
$$


The total carbon content as predicted in the above equations amounts to $79.5 \mathrm{wt} . \%$, while the experimentally measured result was $80.15 \mathrm{wt} . \%$. Similarly, the calculated fluorine content accounts for $13.8 \mathrm{wt} . \%$, while 15.69 wt.\% was not attributed to carbon, nitrogen, hydrogen, or sulfur in the experimental results. The remaining unattributed mass can be attributed to oxygen and other atoms arising from nGTrz, as well as any newly added oxygen functionalities, for instance from any hydrolyzed chlorines of nGTrz.

Using these same methods, the theoretically expected content of nGTrz-Oct should be 92.5 wt.\% Carbon, 1.4 wt.\% Hydrogen, 2.2 wt.\% Nitrogen, and 2.5 wt.\% Sulfur. These values match quite closely to the experimentally measured results from Table 1 in the main text. 


\section{Photothermal Efficiency}

Photothermal efficiency was calculated based on the energy input of the laser (derived from laser power, spot size, and irradiation time), concentration of graphene nanomaterial, and energy transferred to the solution (determined by specific heat capacity, volume, and temperature change), as shown below. ${ }^{1}$

$$
\begin{gathered}
E_{\text {laser }}=0.5 \mathrm{Wcm} \mathrm{cm}^{2} \times \text { Area }\left(5 \mathrm{~mm} \text { spot, } 0.196 \mathrm{~cm}^{2}\right) \times \text { Duration }(240 \mathrm{~s})=23.55 \mathrm{~J} \\
C_{n G}=10 \mu \mathrm{g} / \mathrm{mL} \quad C_{n G T r z}=10 \mu \mathrm{g} / \mathrm{mL}(90 \% \mathrm{nG}) \quad C_{n G T r z-P F}=10 \mu \mathrm{g} / \mathrm{mL}(75 \% \mathrm{nG}) \\
E_{\text {water }}=4.2 \mathrm{~J} \mathrm{~mL}^{-1} \mathrm{~K}^{-1} \times 0.2 \mathrm{~mL} \times \Delta \mathrm{T}
\end{gathered}
$$

Per the photothermal heating results in Figure 4a:

$$
\begin{gathered}
\Delta T_{n G}=52.3 \mathrm{~K} \quad \Delta T_{n G T r z}=50.4 \mathrm{~K} \quad \Delta T_{n G T r z-P F}=43.7 \mathrm{~K} \\
\eta_{x}=E_{\text {water }} / C_{x} / E_{\text {laser }}
\end{gathered}
$$

Thus,

$$
\begin{gathered}
\eta_{n G}=0.23 \mu g^{-1} \\
\eta_{n G T r z}=0.21 \mu g^{-1}\left(\text { normalized to nG content }: 0.23 \mu g^{-1}\right) \\
\eta_{n G T r z-P F}=0.19 \mu g^{-1}\left(\text { normalized to } n G \text { content }: 0.24 \mu g^{-1}\right)
\end{gathered}
$$




\section{Raman Spectroscopy}

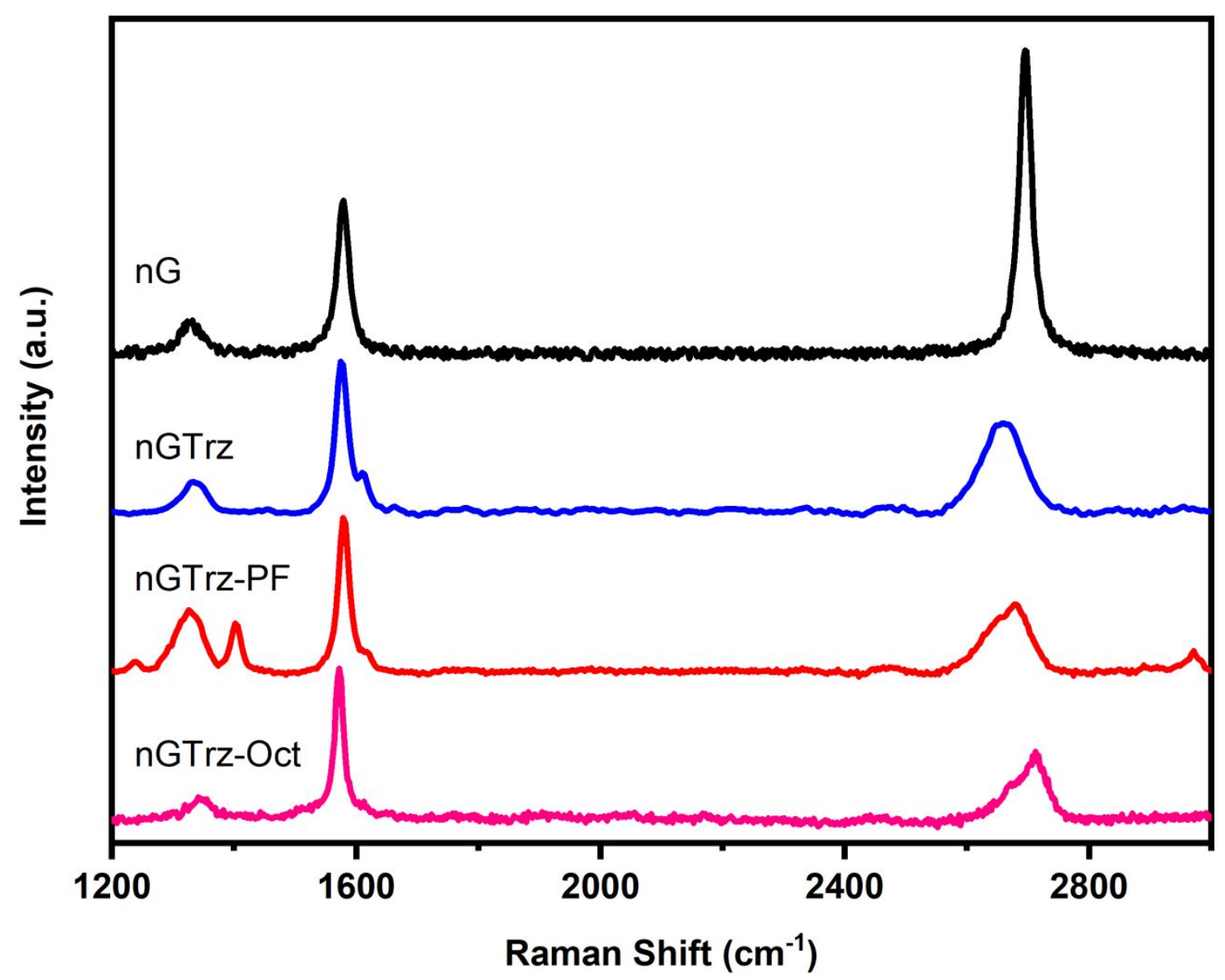

Figure S1. Raman spectra of $\mathrm{nG}$ starting material, functionalized nGTrz, and post-modified nGTrz-PF and nGTrz-Oct sheets. The D $\left(\sim 1325 \mathrm{~cm}^{-1}\right), \mathrm{G}\left(\sim 1580 \mathrm{~cm}^{-1}\right)$, and 2D $\left(\sim 2680 \mathrm{~cm}^{-1}\right)$ bands of graphene are present in all samples.

As previously reported, the $\mathrm{D} / \mathrm{G}$ ratio of $\mathrm{nG}$ and $\mathrm{nGTrz}$ are unchanged. The new shoulder on the $\mathrm{G}$ band, visible in nGTrz as well as nGTrz-PF, arises from heteroatoms in the graphene sheets in this case, the nitrogen conjugating Trz to $\mathrm{nG}$. The change in the 2D signals around $2700 \mathrm{~cm}^{-1}$ arise from the quasi-amorphous distortions of the graphene crystal structure after conjugation of the triazine groups. ${ }^{2}$

New bands arising from the perfluorinated chains can be seen shortly above $1200 \mathrm{~cm}^{-1}$ and at about $1400 \mathrm{~cm}^{-1}$, with a possible third signal overlapping with the D band. In contrast, this signal is not present in nGTrz-Oct, further supporting the attribution to the perfluorinated sections. 


\section{NMR Analysis and expanded results}

As discussed in the main text, the chlorination of both 1-decanol and 3-phenyl-1-propanol could be easily followed by NMR. In the case of 1-decanol, there arise a series of peaks at upwards of $4.1 \mathrm{ppm}$ which can be attributed to hydrogen atoms adjacent to glyoxylate. At $3.5 \mathrm{ppm}$, chlorine-adjacent hydrogens appear, while multiple signals between 0.8 and $1.8 \mathrm{ppm}$ arise from the remaining alkyl hydrogens. Meanwhile, the alcohol hydrogen of 1-decanol gives rise to a singlet at $1.84 \mathrm{ppm} .^{2}$ While the alcohol-adjacent hydrogens could overlap with those of the chlorinated product, this can be appropriately normalized with regards to the -OH signal. It is worth noting that firstly, oxalyl chloride is used in sufficient quantities that nonchlorinated alcohols are extremely likely to react to form chloroglyoxalylates or bisoxalylesters. Indeed, the NMR spectra showed almost no measurable signal attributable to the -OH signal of 1-decanol. In the case of 3-phenyl-1-propanol, a similar set of signals arise, with a change in the alkyl region due to the short propyl chain and a new aromatic signal that is, thankfully, essentially unchanged before or after chlorination.

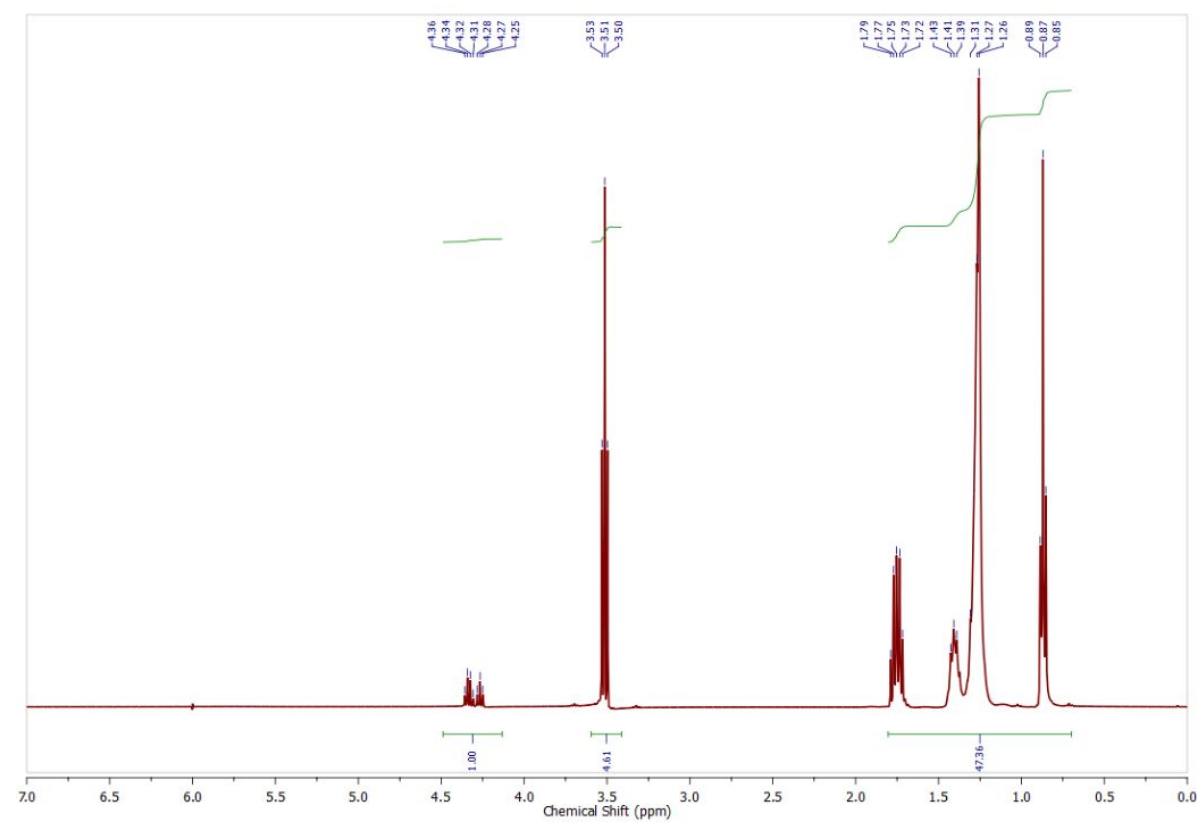

Figure S2. Full ${ }^{1} \mathrm{H}$ NMR spectrum of 1-decanol chlorination products after reaction with nGTrzPF-fTPP under NIR irradiation.

Integrals from the respective signals are presented below for each alcohol across 10 repeated reaction cycles (Table S1, Table S2), along with the calculated yield of their respective chlorides. Given the negligible amount of unconverted alcohol in all reactions (remaining oxalyl chloride is extremely reactive with alcohols), the yield was calculated simply as follows:

$$
\text { Yield }=\frac{\text { Cl integral }}{\text { Clintegral }+ \text { Glyoxylate integral }}
$$




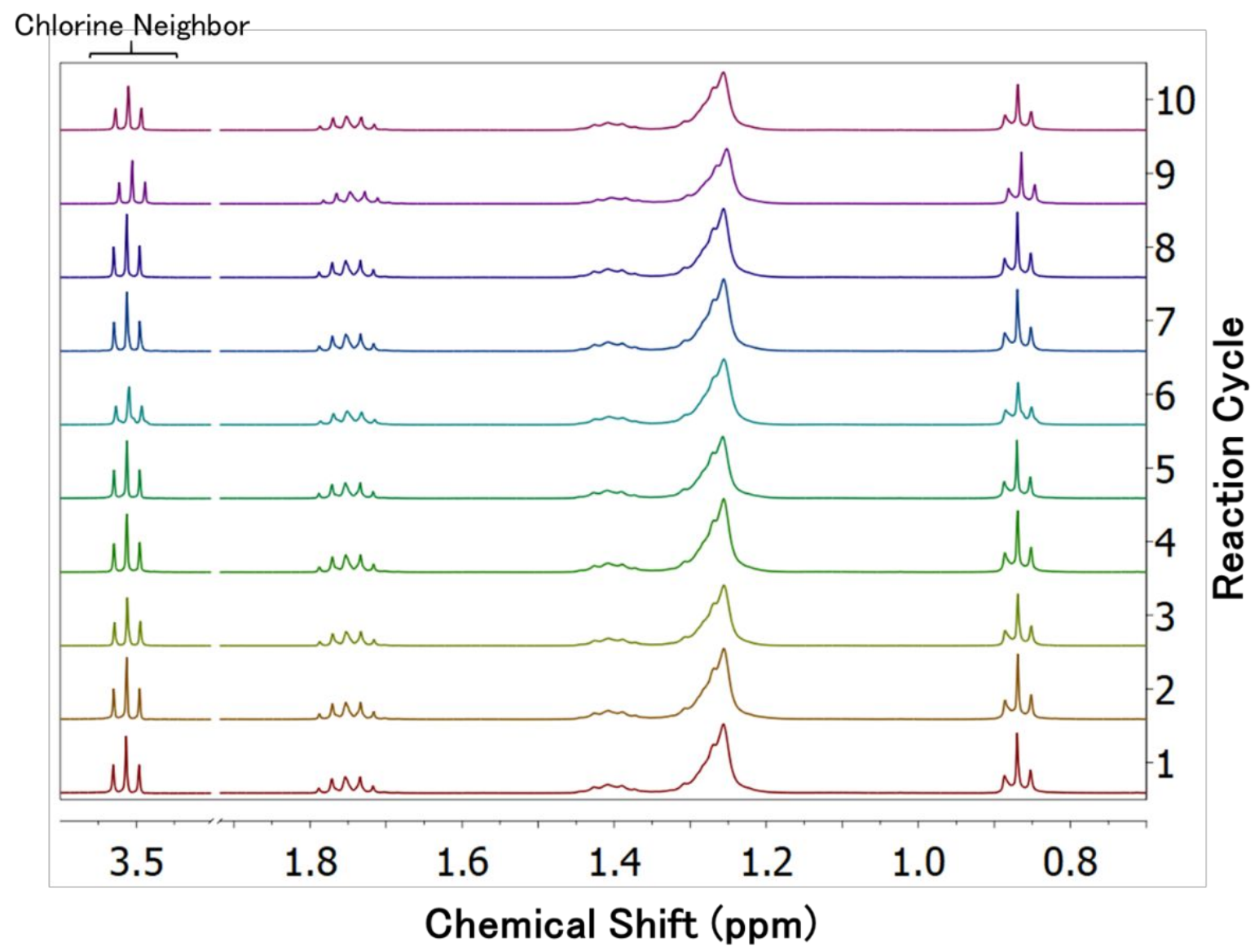

Figure S3. Abbreviated NMR spectra for 1-decanol chlorination products across 10 reaction cycles.

Table S1. NMR Integrals and Calculated Reaction Yields for 1-Decanol Chlorination

\begin{tabular}{c|rrr} 
Reaction \# & Cl Integral & Glyoxylate Integral & Yield \\
\hline 1 & 5.54 & 0.98 & 86 \\
2 & 4.38 & 0.86 & 74 \\
3 & 5.92 & 0.99 & 87 \\
4 & 6.29 & 1.00 & 87 \\
5 & 8.06 & 0.93 & 87 \\
6 & 6.35 & 0.99 & 90 \\
7 & 6.28 & 0.97 & 86 \\
8 & 6.72 & 1.01 & 86 \\
9 & 2.75 & 0.99 & 84 \\
10 & 5.53 & 0.89 & 85
\end{tabular}




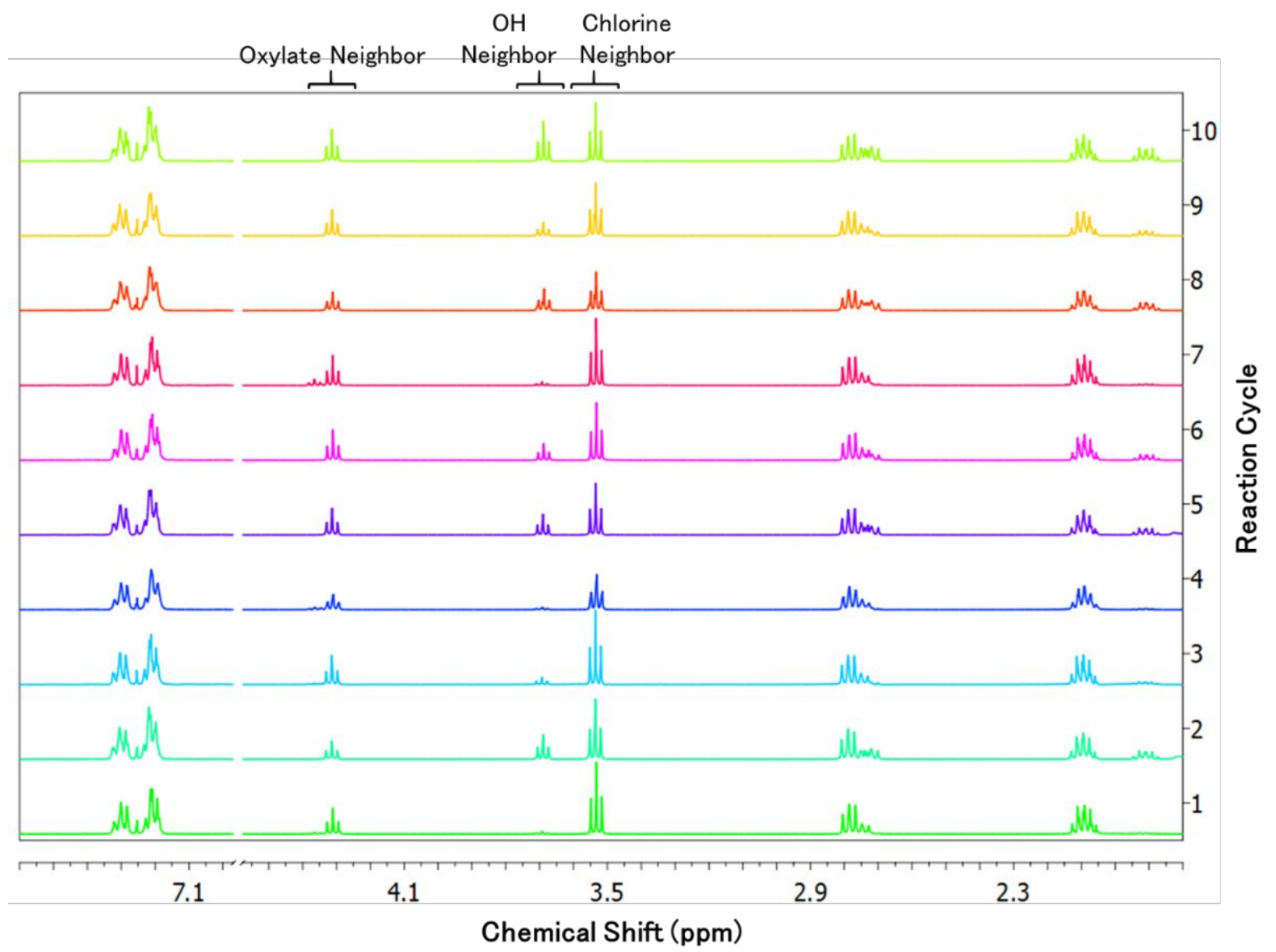

Figure S4. Abbreviated NMR spectra for 3-phenyl-1-propanol chlorination products across 10 reaction cycles.

Table S2. NMR Integrals and Calculated Reaction Yields for 3-Phenyl-1-propanol Chlorination

\begin{tabular}{c|rrr} 
Reaction \# & Cl Integral & Glyoxylate Integral & Yield \\
\hline 1 & 0.26 & 0.14 & 65 \\
2 & 0.22 & 0.18 & 55 \\
3 & 0.25 & 0.16 & 61 \\
4 & 1.60 & 1.19 & 57 \\
5 & 1.67 & 1.71 & 49 \\
6 & 1.61 & 1.50 & 52 \\
7 & 1.52 & 1.11 & 58 \\
8 & 0.18 & 0.21 & 46 \\
9 & 1.70 & 1.47 & 54 \\
10 & 1.54 & 2.12 & 42
\end{tabular}




\section{References}

1. Liu, G.; Zhao, P.; Liu, N.; Yoshino, F.; Qin, H.; Zou, Y.; Shi, S.; Amano, T.; Aguilar Cosme, J. R.; Nagano, Y.; Tamiaki, H.; Komatsu, N., Photosensitizer and anticancer drug-loaded 2D nanosheet: Preparation, stability and anticancer property. 2D Materials 2019, 6, 045035.

2. Denton, R. M.; An, J.; Adeniran, B., Phosphine oxide-catalysed chlorination reactions of alcohols under Appel conditions. Chemical Communications 2010, 46, 3025-3027. 